Projets

de paysage

\section{Projets de paysage}

Revue scientifique sur la conception et l'aménagement de l'espace

$5 \mid 2011$

Le jardin et ses rapports à l'art. Encadrer, décadrer, recadrer

\title{
Une architecture de cadres
}

An Architecture of Frames

Laurence Kimmel

\section{(2) OpenEdition}

\section{Journals}

\section{Édition électronique}

URL : https://journals.openedition.org/paysage/21408

DOI : $10.4000 /$ paysage. 21408

ISSN : 1969-6124

\section{Éditeur :}

École nationale supérieure du paysage de Versailles-Marseille, Institut national des sciences appliquées Centre Val de Loire - École de la nature et du paysage, École nationale supérieure d'architecture et de paysage de Bordeaux, École nationale supérieure d'architecture et de paysage de Lille, Agrocampus Angers

\section{Référence électronique}

Laurence Kimmel, « Une architecture de cadres », Projets de paysage [En ligne], 5 | 2011, mis en ligne le 13 janvier 2011, consulté le 16 octobre 2021. URL : http://journals.openedition.org/paysage/21408 ; DOI : https://doi.org/10.4000/paysage.21408

Ce document a été généré automatiquement le 16 octobre 2021.

\section{c) () $\Theta$}

La revue Projets de paysage est mise à disposition selon les termes de la Licence Creative Commons Attribution - Pas d'Utilisation Commerciale - Pas de Modification 4.0 International. 


\title{
Une architecture de cadres
}

\author{
An Architecture of Frames
}

\section{Laurence Kimmel}

1 Certains jardins contemporains sont conçus comme des «jardins philosophiques». Pour le Jardin du souvenir (1999) à Duisburg, Dani Karavan a ancré son travail sur le thème de la mémoire, comme dans plusieurs de ses projets. Cette signification latente, par les symboles utilisés, s'allie au niveau perceptif, dans le cas de l'une des sculptures, à de multiples cadres sur l'environnement. Le sens de ces cadres peut être rapproché de la philosophie de l'histoire de Walter Benjamin, qui est une référence profonde de Dani Karavan.

2 Dans le domaine du paysage, le cadre a une fonction classique d'artialisation de la vue que l'on a à travers celui-ci. Cette culture perceptive est exposée notamment par Alain Roger dans son ouvrage Court Traité du paysage (1997). Le cadre transforme la vue d'un paysage non esthétisé (dans la limite de cette possibilité) en paysage sur lequel le regard s'arrête, fait une pause, en portant une attention aux éléments cadrés et à leur agencement sur une "toile virtuelle», telle que théorisée par Brunelleschi. Ce phénomène simple, mais qui revêt toutefois des caractéristiques variables suivant les périodes, peut être considéré comme un phénomène classique de notre appréhension du paysage et du monde. Le cadre, par ce processus de transformation du champ visuel en représentation, crée un jeu perceptif et imaginaire sur les distances. Parmi les exemples classiques, notons la présence d'éléments latéraux et, a fortiori, une série d'éléments qui «éloignent» le paysage perçu en arrière-plan. Autre principe: les éléments d'une zone cadrée ont une distance qu'il est difficile d'apprécier, et ainsi le cadre augmente notre tendance à une "perception imageante» du paysage. Les éléments semblent donc "ramenés» sur un même plan. Nous perdons l'échelle par manque de continuité du proche au lointain, par manque de repères latéraux, qui sont rendus «flottants » par le cadre. Plus que ce jeu, le dispositif du cadre, par système de visée, crée une échelle imaginaire ${ }^{1}$ : l'infini, pour Morris, dans l'installation Observatory (1977) et, ici aussi, dans le cadrage du ciel en partie haute.

3 La question qui anime le présent article est celle de la multiplicité des cadres dans notre champ de vision. Si deux cadres coexistent devant nous, alors le passage à une 
représentation dans chacun des cadres peut être alterné ou simultané, et dans tous les cas des liens de forme et de sens sont recherchés entre les deux « représentations ». Au minimum, on considère les deux ensembles cadrés en évaluant la possibilité de la coexistence spatiale des deux ensembles, voire en imaginant les liens spatiaux plus ou moins précis et conscients entre les deux vues. Une dialectique s'instaure entre deux représentations qui tendent vers le statut d' "images". Notre regard a tendance à «faire un saut » d'un cadre à un autre, même si une liberté générale d'appréhension demeure : les cadres sont un moyen efficace de structuration du champ visuel, un début d'architecture qui s'interpose entre le moi et l'environnement, et institue le monde comme représentation.

4 L'œuvre étudiée est située à Duisburg, dans la Ruhr (ancienne zone industrielle de l'Allemagne). En 1999, Dani Karavan, sculpteur israélien né en 1930 à Tel Aviv et vivant entre Tel Aviv, Paris et Florence, y termine l'aménagement du Jardin du souvenir, dans la zone du « port intérieur », dans le cadre d'un projet urbain dont l'architecte en chef est Norman Foster. Karavan est connu pour avoir réalisé entre autres le Monument du Negev ${ }^{2}$ (1963-1968) et l'Axe majeur de Cergy-Pontoise (1980- ). Il a réalisé des sculptures environnementales (site specific) symboliques, dans une démarche et une pensée optimistes quant à leur impact social et politique potentiel. Dans le Monument du Negev, l'ensemble très architecturé crée des vues cadrées sur le lointain, qui coexistent dans le parcours avec des lieux proches. Cet ensemble génère une poétique de cette coexistence des échelles et de notre manière d'habiter l'espace proche et lointain.

Figure 1. Dani Karavan, Jardin du souvenir, Duisburg, 1999

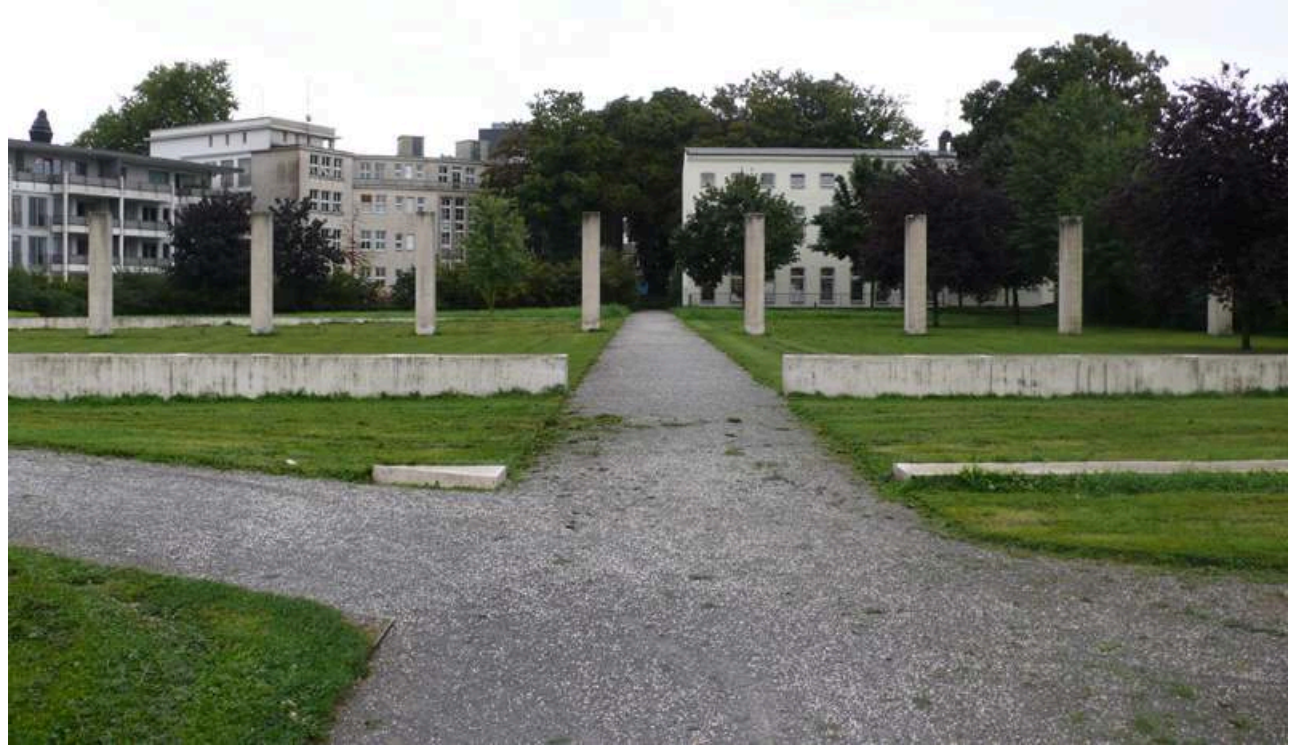

Dans le cas du Jardin du souvenir, l'installation n'est pas centrée mais multicentrée (deux tours en béton, des restes de charpentes métalliques ou en béton, des chemins en ligne droite qui traversent le site). Aucune architecture ne nous "enferme» dans un lieu circonscrit. L'architecture qui préexistait à l'aménagement du jardin (des bâtiments industriels d'après-guerre assez banals) a été en partie soustraite, démolie par Karavan (le geste du sculpteur est d'ailleurs parfois celui d'une soustraction de la matière), de 
manière à obtenir cet "espace ouvert» où ne subsistent que des fragments d'architectures et aussi des ruines entassées à quelques endroits.

6 Ce qui nous intéresse tout particulièrement réside dans le fait qu'à deux reprises ces « restes » de bâtiments sont des structures. La structure en béton d'un bâtiment central et de grande ampleur crée un système linéaire de cadres, dans les trois dimensions. Karavan, que nous avons interviewé pour cette recherche, confirme cette intention, généralisable à l'ensemble de son travail plastique ${ }^{3}$. Par contraste avec les tours/cages d'escalier qui sont des sculptures centrées ayant une caractéristique d'objet, la nonobjectivité et la transparence des cadres mettent ces derniers d'autant plus en connexion avec les échelles de la ville. Dans la démarche de Karavan, ce travail sur les cadres n'est pas à concevoir de manière purement abstraite, mais comme œuvrant à une prise de conscience de l'espace et du temps, notamment de la mémoire historique collective.

Figure 2. Dani Karavan, Jardin du souvenir, Duisburg, 1999

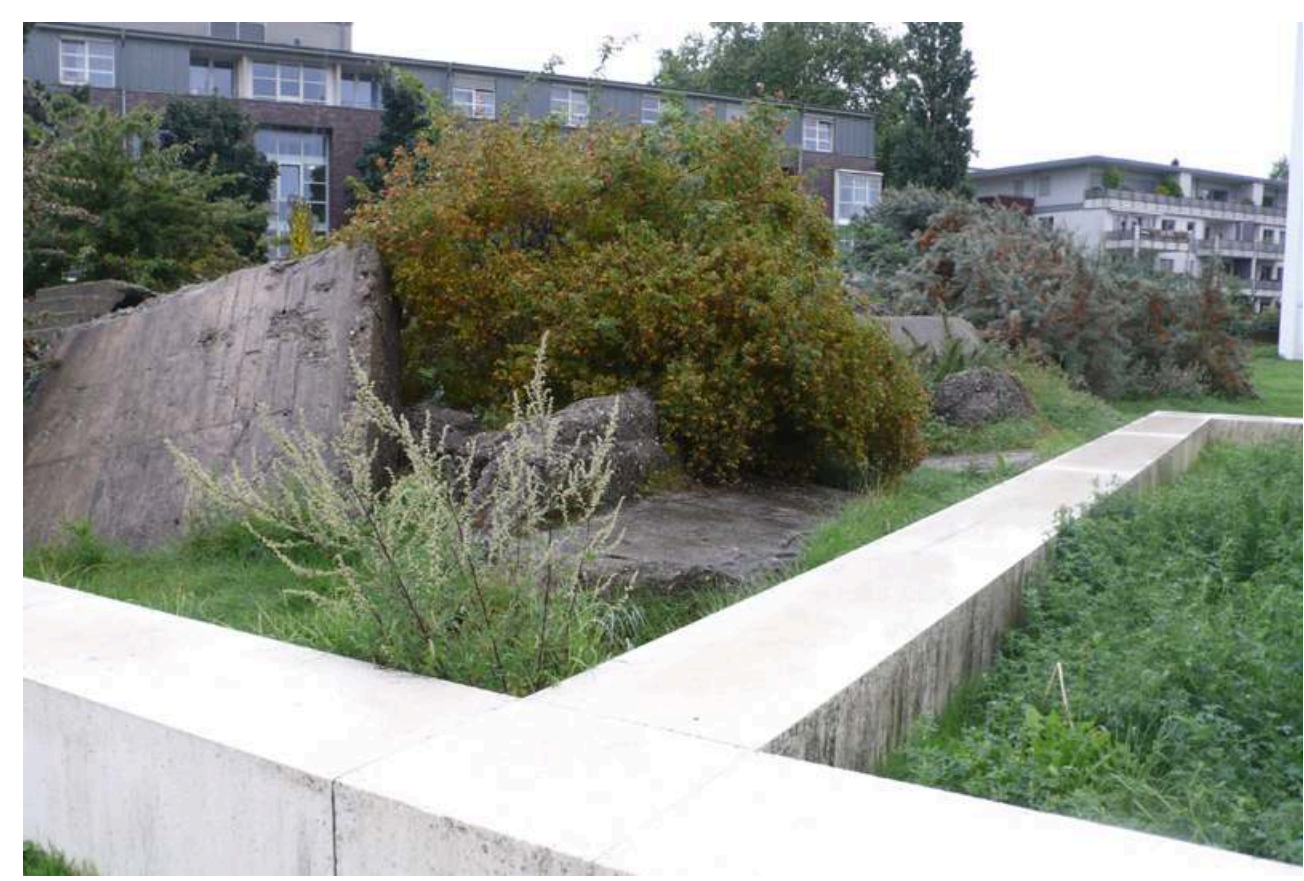

7 Les «ruines» déposées par Karavan recréent un paysage familier de la fin de la Seconde Guerre mondiale, reconstitué "artificiellement", en particulier devant la maison de retraite, dans un geste quelque peu provocateur. Ces restes s'apparentent aux «rebus de l'histoire» dont parle Walter Benjamin. Les ruines sont entreposées dans une des zones cadrées au sol par les chemins et également au fond d'une impasse, celle du Philosophenweg, comme si Karavan avait fait un tri, sur le modèle de la méthode d'historien de Benjamin: "D'un côté se trouve la partie "féconde", "pleine d'avenir", "vivante", "positive", de l'autre la partie inutile, arriérée et morte de cette époque.» (Benjamin, 2009, p. 475.) 
Figure 3. Dani Karavan, Jardin du souvenir, Duisburg, 1999

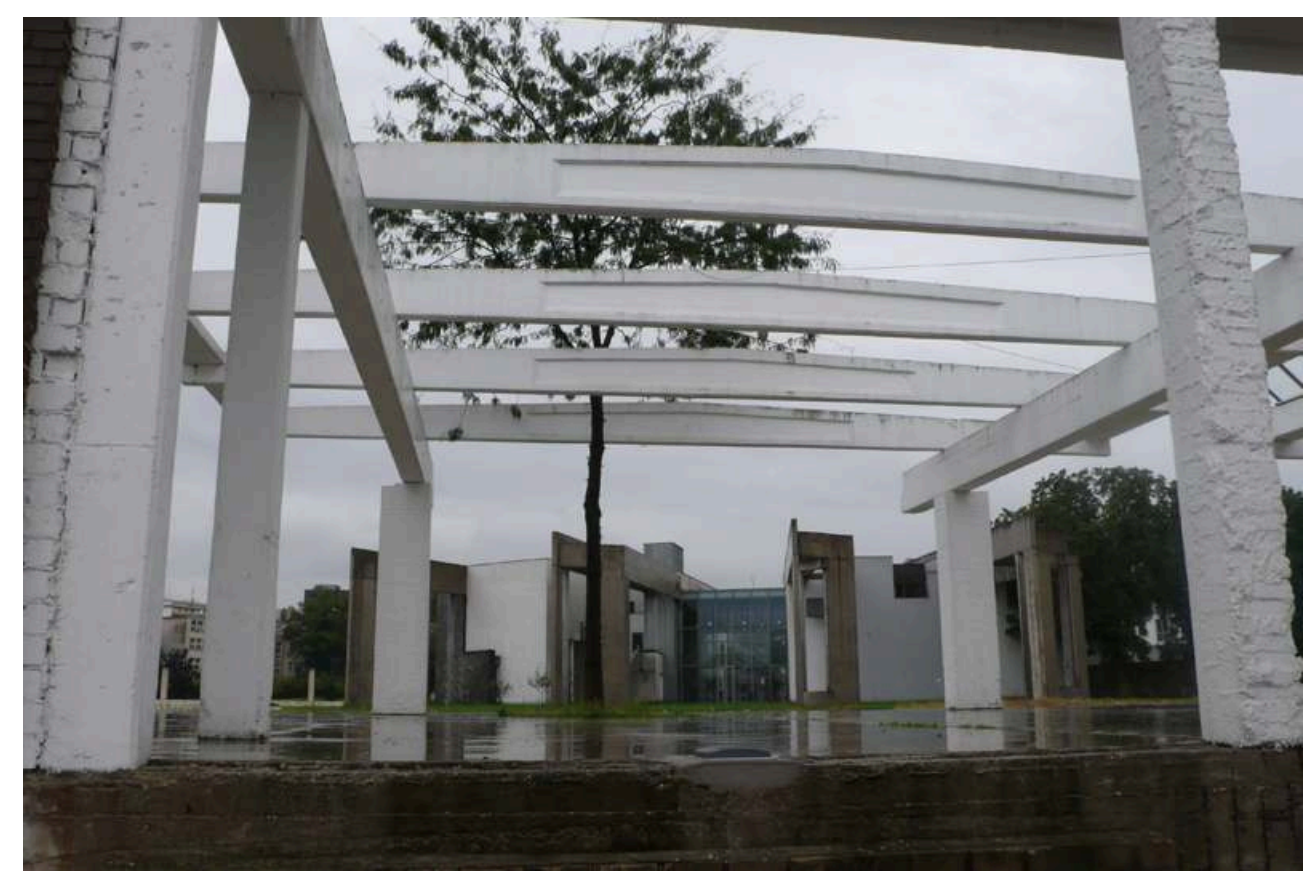

8 Ainsi les cadres, même s'ils sont entièrement peints en blanc, ce qui en augmente l'abstraction ${ }^{4}$, sont inexorablement d'un blanc qui porte une signification de conservation, de mémoire historique, «comme un paysage primitif pétrifié » (Benjamin, 2009, p. 478, citant Adorno à propos de l'allégorie), comme une «image noir et blanc qui présente déjà sa propre mémoire ${ }^{5}$ ». L'aménagement de ces cadres, au sol (les chemins rectilignes) et en hauteur (la structure poteau-poutre du bâtiment en béton), ouvre l'ensemble des éléments environnants à cet imaginaire des stratifications historiques. Les cadres "spatiaux " créent un jeu entre les différents éléments représentés, en intégrant les éléments périphériques du jardin. Le sens temporel de ces derniers est donc adjoint et mis en relation avec le sens temporel du cycle de construction (murets et chemins), de transformation (bâtiments), de destruction (façades) orchestré par Karavan. En particulier, les ruines de l'ancienne fortification, extérieures au jardin, paraissent participer de l'ensemble. Notons que, même si la vision imageante créée par les cadres est plus effective lorsque les bords sont à angle droit, en vision de face, ce statut de l'image persiste en vision de biais. Et pour les cadres autres que rectangulaires, de face ou de biais, on peut dire que ce statut perdure, dans une moindre mesure. 
Figure 4. Dani Karavan, Jardin du Souvenir, Duisburg, 1999

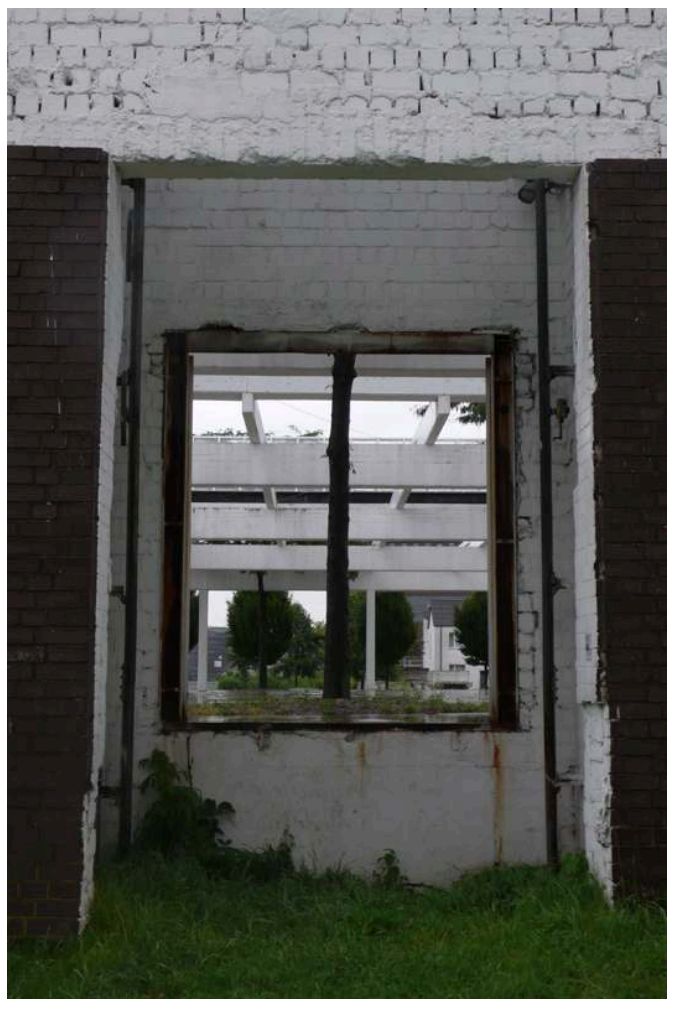

Figure 5. Dani Karavan, Jardin du Souvenir, Duisburg, 1999

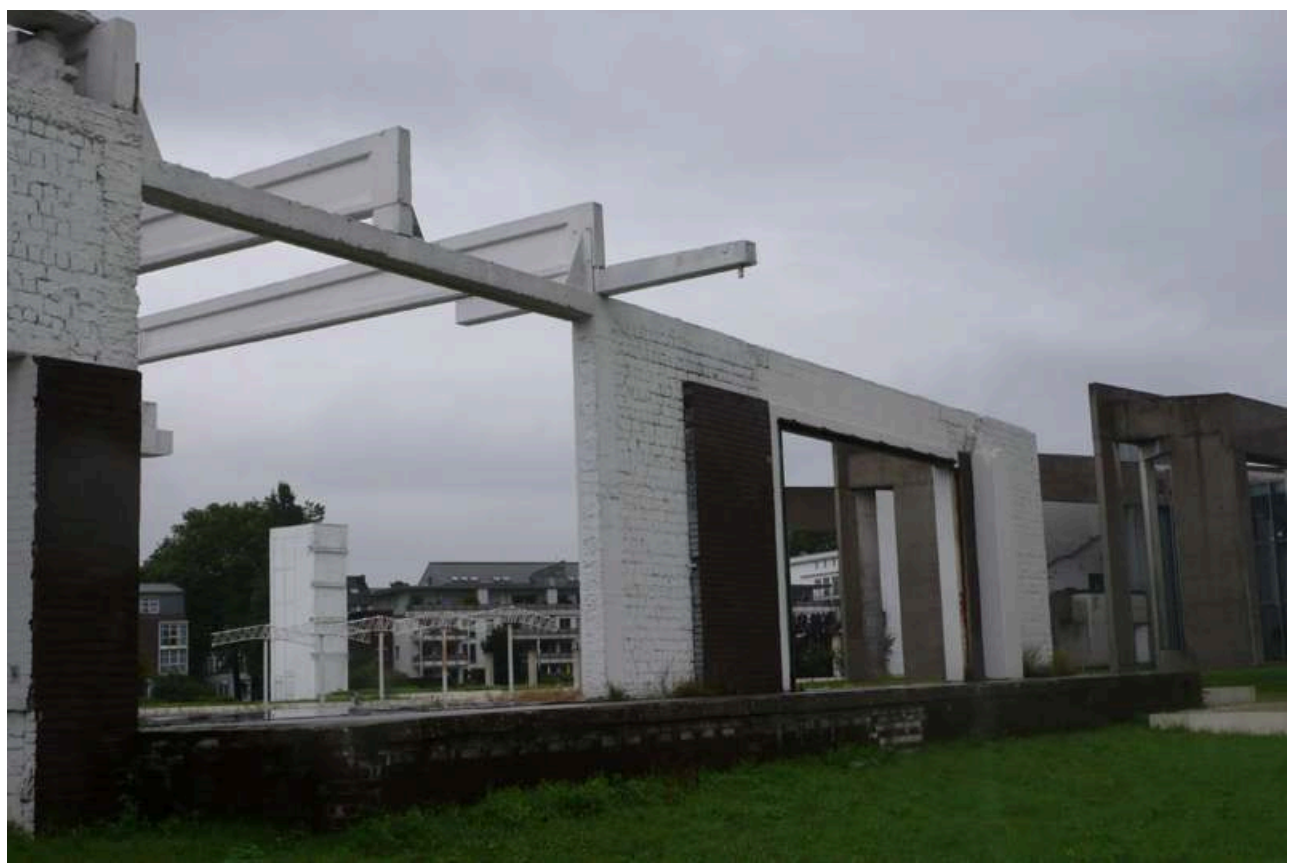


Figure 6. Dani Karavan, Jardin du Souvenir, Duisburg, 1999

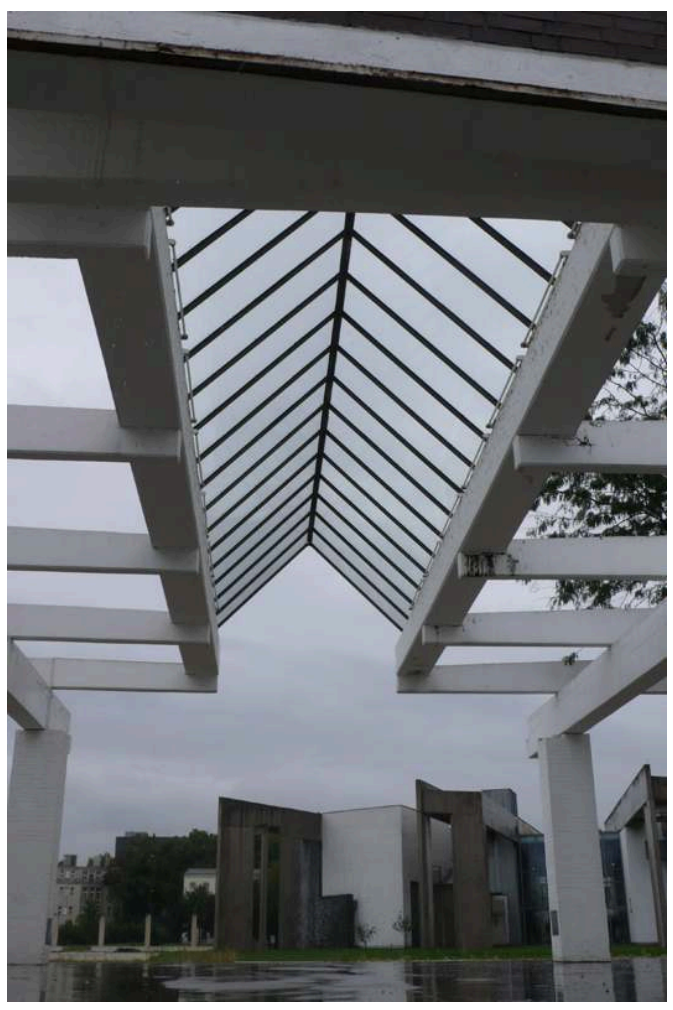

Figure 7. Dani Karavan, Jardin du Souvenir, Duisburg, 1999

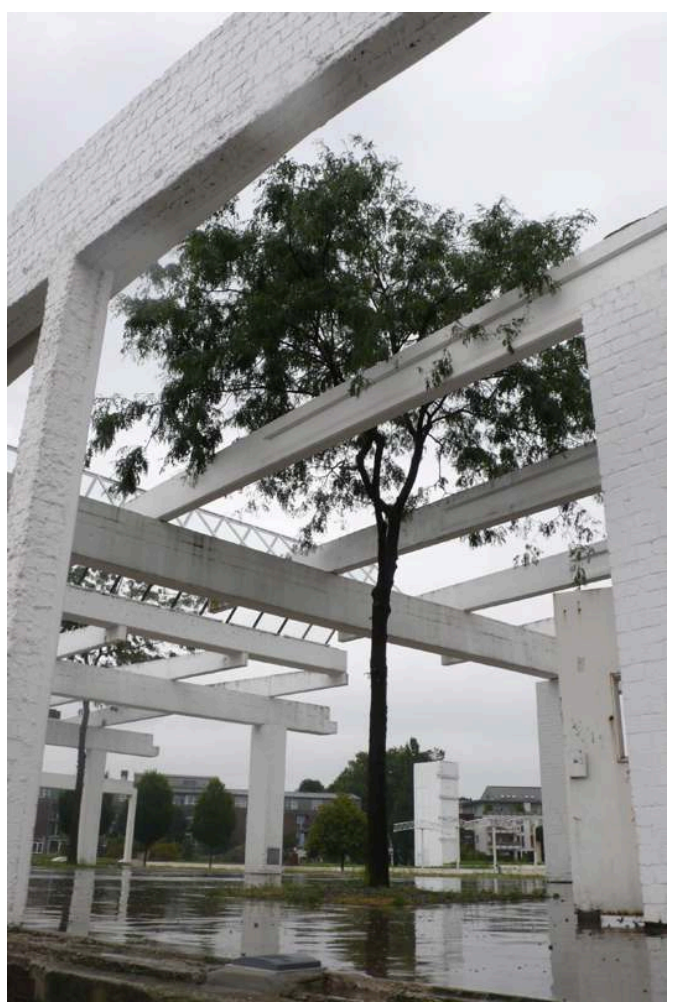

9 En complément du témoignage de Karavan sur le rôle des structures du bâtiment comme cadres, on observe que le bâtiment du Gemeindezentrum (1999) de l'architecte 
Zvi Hecker présente également des cadres en béton, comme des prolongements du corps principal. Les cinq branches sont comme cinq doigts qui «avancent» dans le jardin, créant une imbrication entre ces derniers, comme "un livre ouvert ", selon Hecker $^{6}$. Ces éléments ont d'après lui une signification symbolique historique (histoire de la ville de Duisburg), puisqu'une branche est orientée vers l'ancienne synagogue de la ville, disparue pendant la Seconde Guerre mondiale. Ces "pages" en trois dimensions, en épaisseur (même pour des angles de vue de biais), présentent donc plutôt des marges et un vide intérieur. Quelle que soit l'orientation, on perçoit une multiplicité de cadres. Ce parti pris accompagne parfaitement l'esthétique du jardin, et les discussions préparatoires entre Karavan et Hecker en début de projet ont participé de la cohérence de l'ensemble?

Figure 8. Dani Karavan, Jardin du Souvenir, Duisburg, 1999

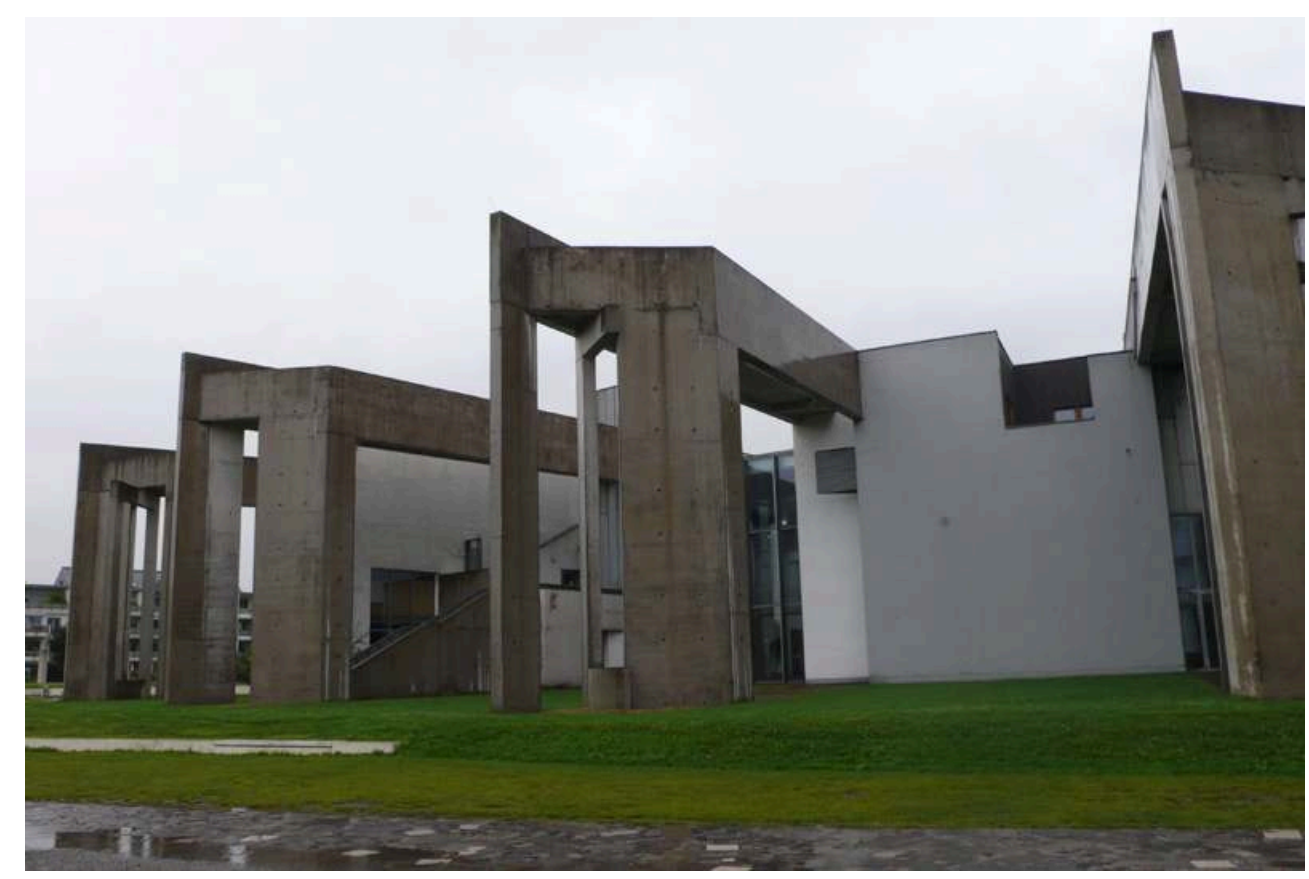


Figure 9. Dani Karavan, Jardin du Souvenir, Duisburg, 1999

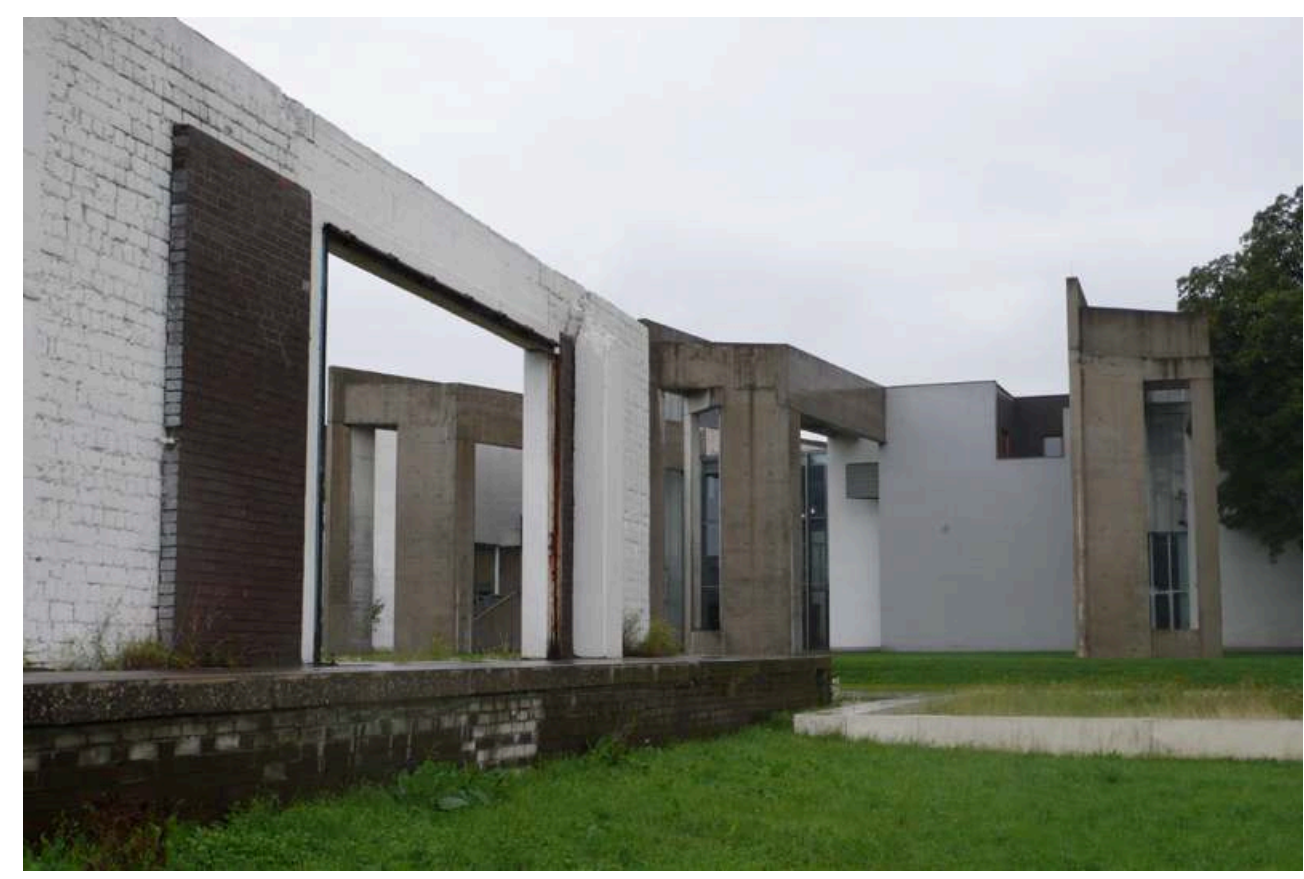

Voyons comment, dans une œuvre antérieure de Karavan, la perception du cadre est empreinte d'une signification temporelle dans l'expérience de son parcours et d'une signification historique par sa fonction de mémorial. Karavan a expérimenté la notion de cadre dans le projet Passages, œuvre dédiée à Walter Benjamin ${ }^{8}$ et réalisée à Portbou, à la frontière franco-espagnole, entre 1991 et 1994. Ce mémorial est composé de trois éléments, situés à trois hauteurs différentes de la falaise surplombant la mer, autour du cimetière où repose le philosophe allemand. Le premier élément est le corridor (ou tunnel). La vision du paysage est cadrée aux deux extrémités par ses parois. Le deuxième élément définit le point de vue depuis la dernière marche d'un promontoire. Le champ de vision est cadré à droite par le mur du cimetière, mais s'élargit néanmoins. Le troisième élément, un piédestal, définit également un point de vue, depuis lequel nous avons une vue dégagée sur la mer. Une attention élargie aux sons et autres sensations de l'environnement surgit, car nous reprenons possession de la grande échelle et de la multiplicité des directions, en rapport et en contrepoint avec le cadrage fort et la direction unique du corridor. Le cadrage par cette architecture ouverte, puis le décadrage qui s'ensuit créent une expérience du paysage plus intense (Kimmel, 2009). 
Figure 10. Dani Karavan, Passages, plateforme, Portbou, 1991-1994

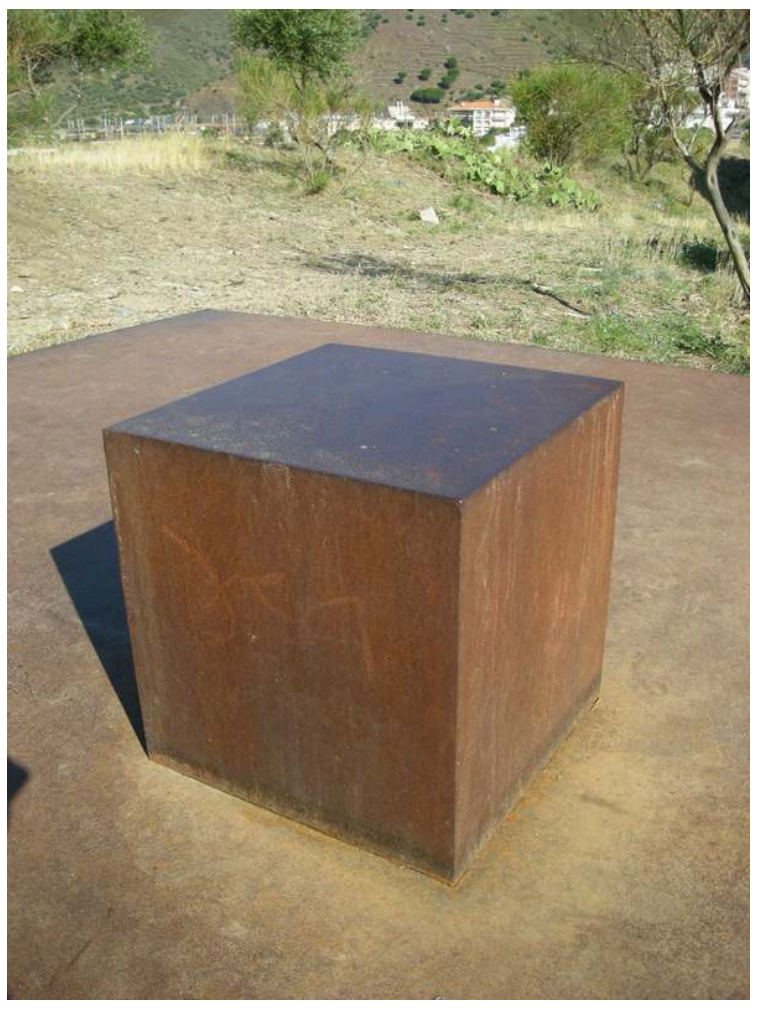

11 Pour préciser ce phénomène, faisons le parcours dans un ordre inhabituel et commençons par la plateforme, un cube en métal posé sur une plaque carrée. L'ensemble apparaît comme un objet qui pourrait relever d'un discours sur l'art minimal, l'objectivité, la présence. Nous verrons plus loin quelle fonction lui a attribuée Karavan. 
Figure 11. Dani Karavan, Passages, corridor, Portbou, 1991-1994

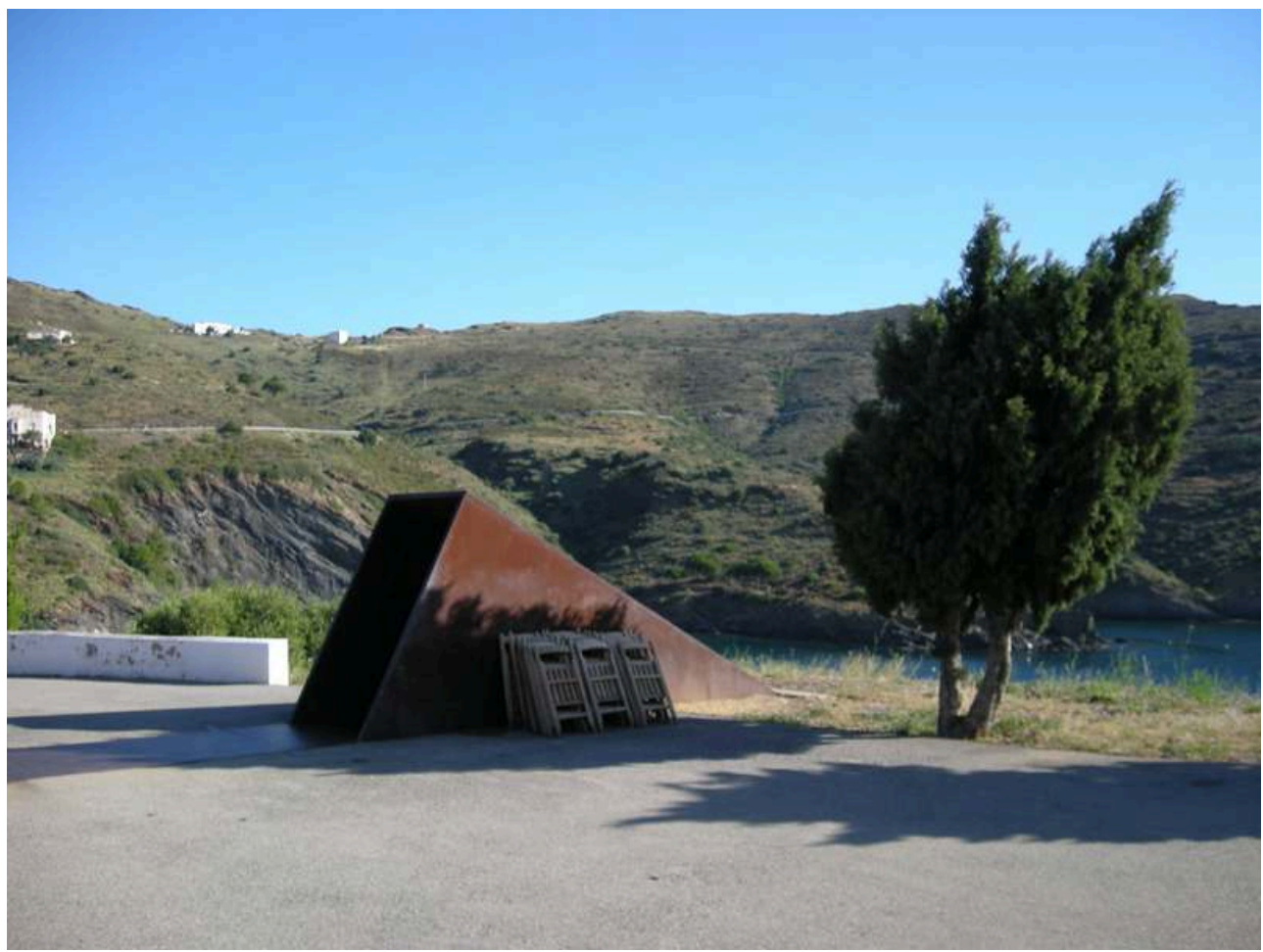

Figure 12. Dani Karavan, Passages, corridor, Portbou, 1991-1994

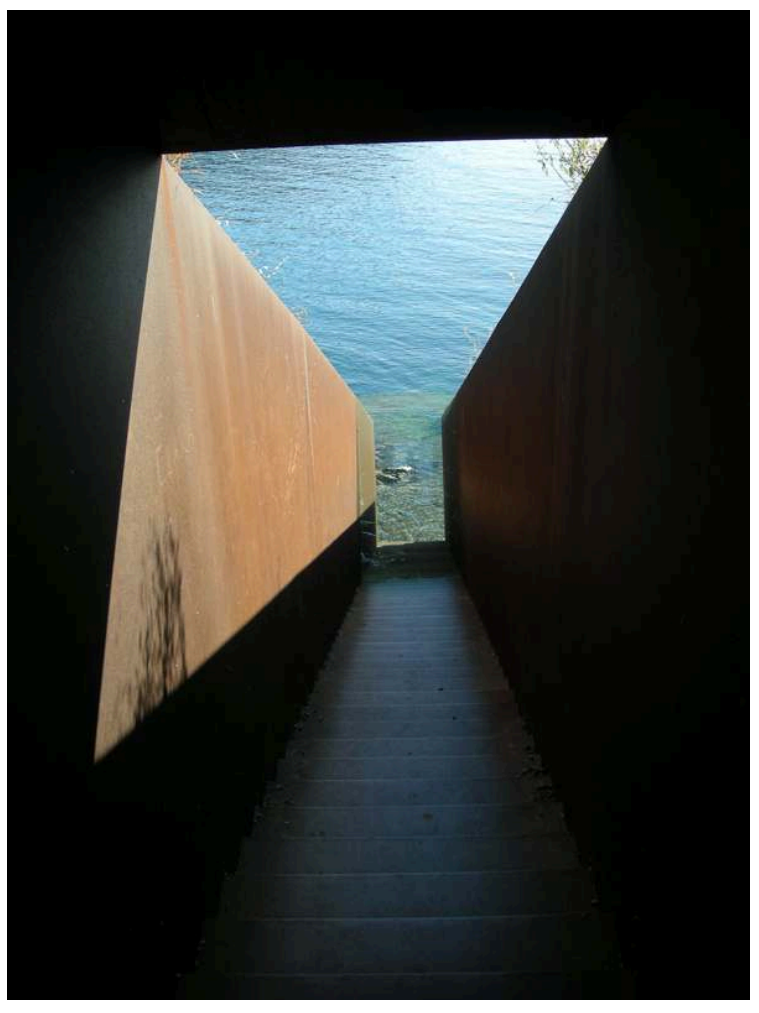

12 Dans ce mémorial en trois étapes, la partie principale, la plus connue, est le corridor. Comme le regard est tourné vers la partie de paysage cadrée, nous perdons le lien corporel au paysage, nous perdons le paysage en quelque sorte. Les cadrages sont 
stricts, même s'il y a une échancrure vers le haut en bas du corridor. Par ailleurs, la vitre nous tient à distance de la fin du corridor tout en maintenant le cadrage. Cette distance avec le paysage le transforme en image, comme une vision cadrée "photographique» du paysage. Même si nous avons une impression de proximité visuelle, à cause de la clarté et du niveau de détail des tourbillons d'eau, nous sommes à distance de cette image. Paradoxalement, cette distance nous amène à éprouver la vue des tourbillons d'eau dans une " proximité sensible ». Cette expérience d'une image qui impressionne, qui crée un «choc », nous donne conscience de la réalité, d'une réalité peut-être liée à la mémoire de Walter Benjamin et des "victimes anonymes" ${ }^{9}$ » de la guerre en général. Ce dernier cherche le développement d'un " contenu métaphysique plus profond »(Benjamin, 1971, p. 102) de l'expérience, loin de limiter la connaissance à une relation empirique entre le sujet et l'objet, et l'expérience créée par l'installation de Karavan semble y tendre.

Figure 13. Dani Karavan, Passages, olivier, Portbou, 1991-1994

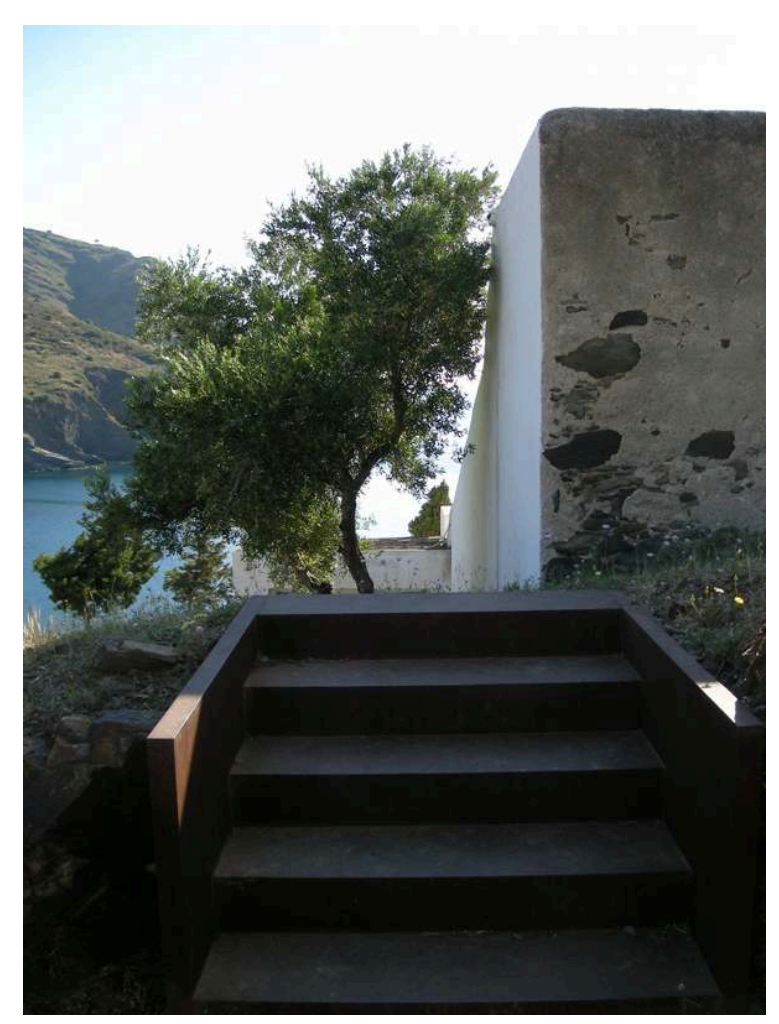

13 L'escalier qui donne la vue sur l'olivier est vécu comme un «demi-cadrage ", en comparaison avec la station précédente. Le mur du cimetière cache la vue sur le lointain, à notre droite. La vision de la partie gauche du champ visuel, avec l'olivier, la vue sur la baie du côté de la ville de Portbou et la vue sur le corridor, est maintenue.

Figure 14. Dani Karavan, Passages, plateforme, Portbou, 1991-1994 


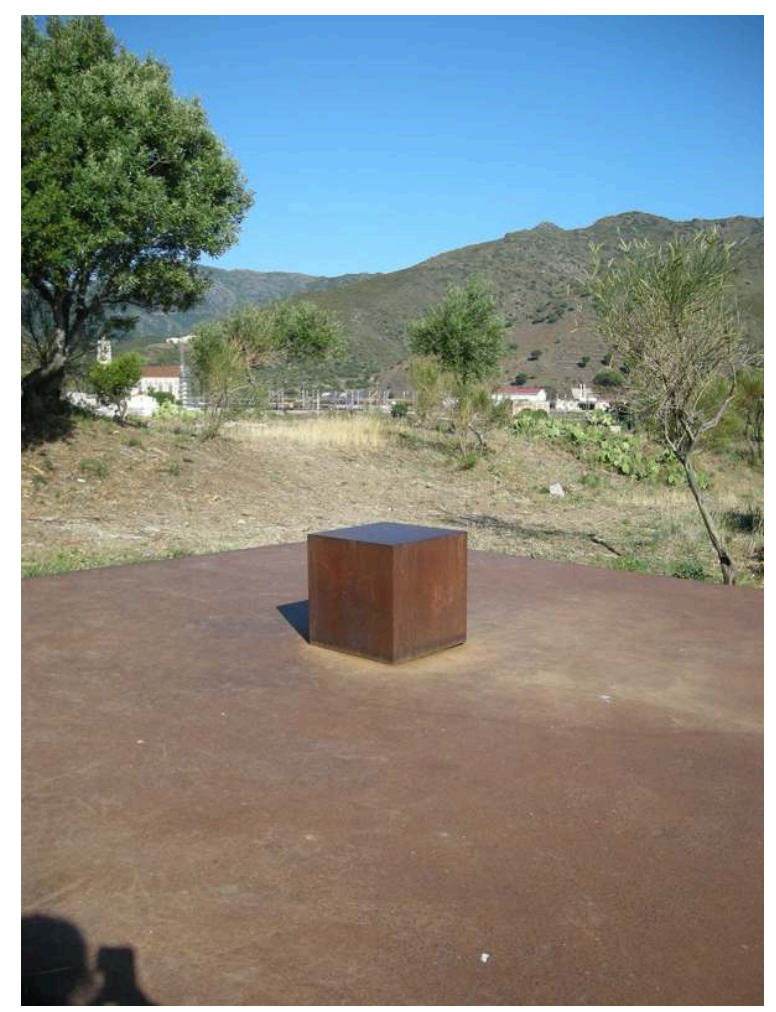

Figure 15. Dani Karavan, Passages, plateforme, Portbou, 1991-1994

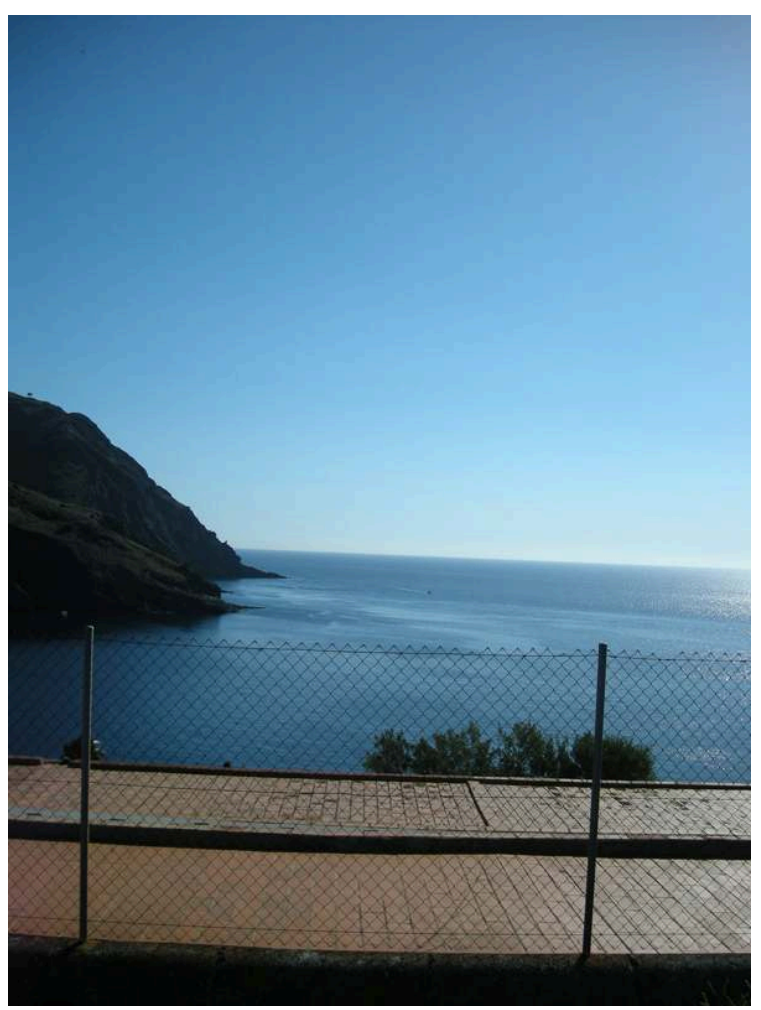

14 Si l'on revient maintenant à la plateforme, celle-ci est vécue comme un décadrage total, car pour Karavan, il s'agit d'un siège à partir duquel on peut observer la mer. On comprend alors l'intention de Karavan de nous ouvrir la vue sur le paysage, en nous incitant à prolonger la contemplation, voire la méditation, en étant assis sur le cube. 
Cette intention peut sembler simple ou classique, mais le regard de l'observateur sera nécessairement influencé par l'expérience préalable des points de vue multiples, du «décadrage»; en somme, par le souvenir des cadres imposés par les précédents éléments et des sensations créées par ceux-ci. Le cadrage peut être un outil de sensation et de pensée, et le « décadrage » peut l'être aussi. Cette succession de stations est une expérience du temps du parcours et de la mémoire des situations précédentes.

Figure 16. Haus Rücker \& Co, Rahmenbau, Kassel, 1977

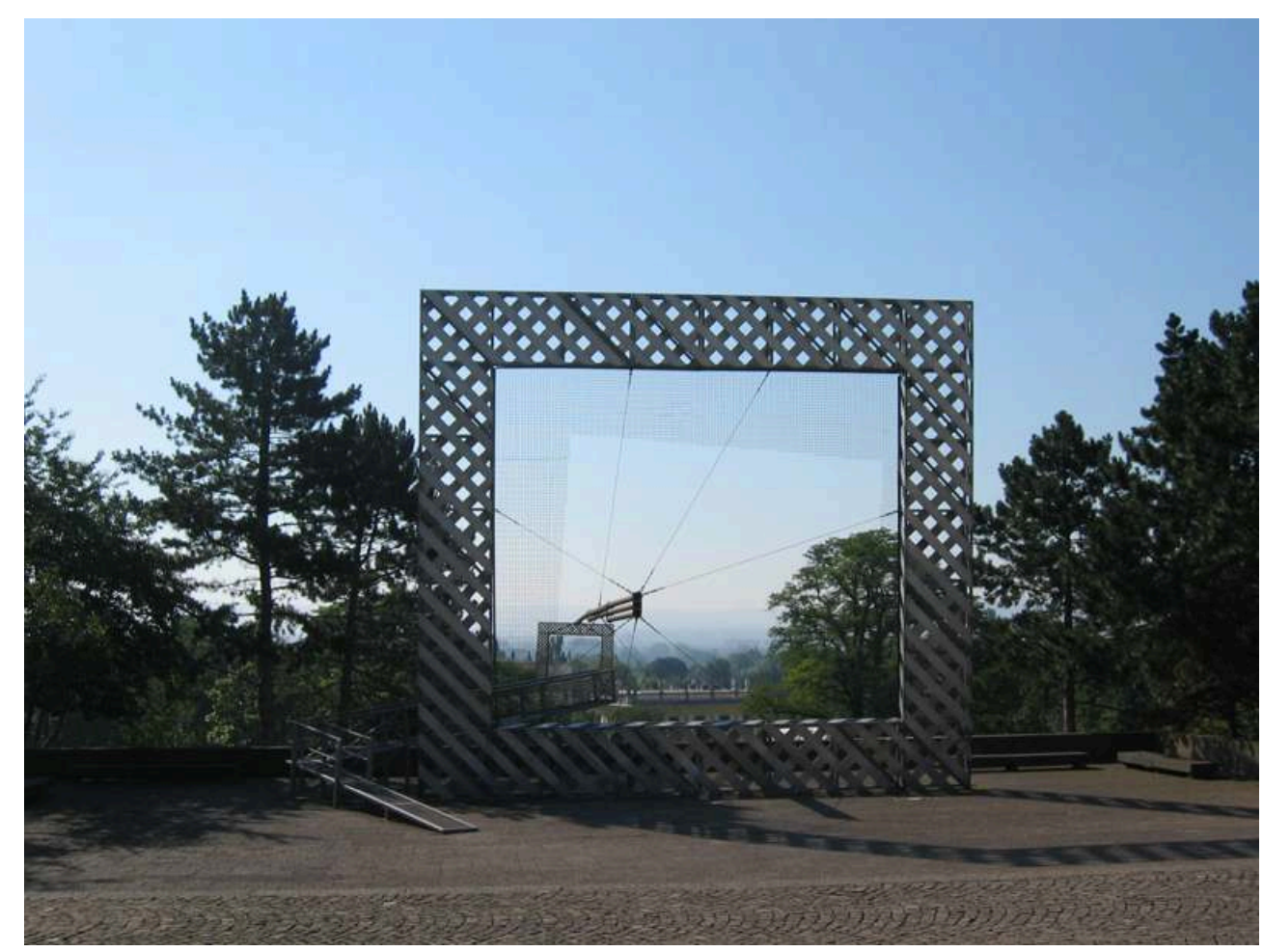




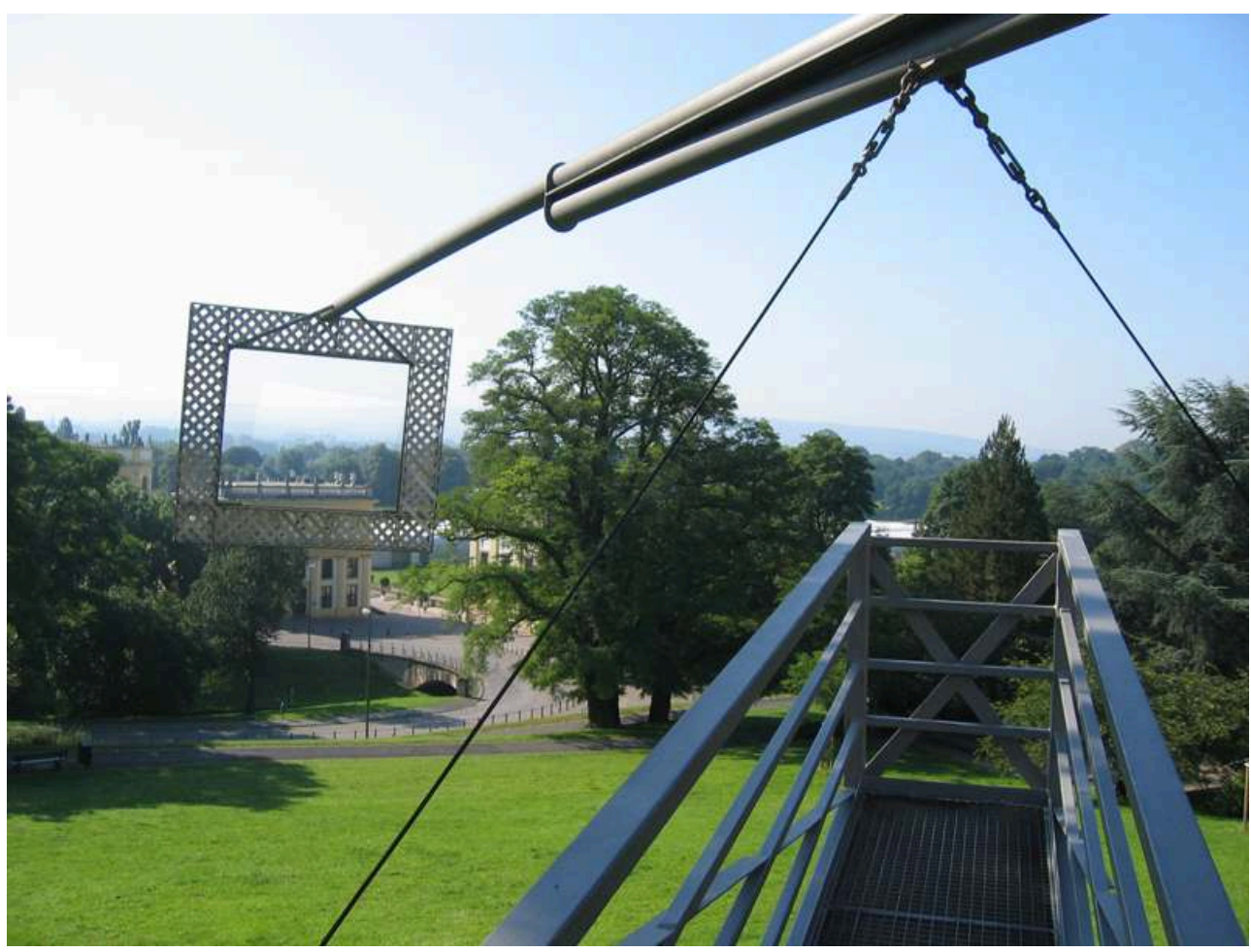

15 La question du cadre comme porteur de signification historique n'est pas nouvelle en 1999. On en trouve un exemple important, conçu par le groupe d'architectes expérimentaux Haus Rücker \& Co, où la multiplicité des cadres n'est pas seulement vécue dans leur succession, mais également dans une coexistence visuelle de ces derniers, un grand et un petit. L'œuvre à laquelle nous faisons référence s'intitule Rahmenbau (Construction de cadre) et a été présentée à la Dokumenta de Kassel, en 1977. Le collectif d'artistes souhaitait que ce cadre soit considéré comme une "architecture pour la conscience et comme complément de l'environnement terrestre ». Le paysage, déjà visible, devient à travers l'œuvre d'art un «champ de conscience élargi ${ }^{10} »$.

Cette conscience est liée à l'histoire, car à cet endroit se trouvait avant 1906 un arc de triomphe. Situé à cet emplacement topographique si particulier de la ville, il attirait autant l'attention que l'installation actuelle. Le Rahmenbau présente les qualités perceptives de l'arche, sans symbole politique et sans la matérialité imposante du monument. La relative neutralité stylistique des cadres et l'ouverture du cadre juste sur ce qu'il y a à voir - le " presque rien de plus » que ce que l'on voit hors dispositif - sont en lien direct avec une mémoire historique et une recherche de conscience des événements passés.

17 Appartenant à la même génération, Dani Karavan a d'une manière similaire vidé les bâtiments de leurs attributs de pouvoir ou de représentation (par exemple leurs façades) pour les réduire à des dispositifs de vision. Contrairement à Haus Rücker \& Co, les structures sont tout de même des témoignages des anciens bâtiments et contribuent au travail sur la mémoire.

Dans le cas du Jardin du souvenir, la multiplicité des cadres, voisins dans la continuité ou imbriqués, transforme la vision, depuis un point de vue proche ou intérieur à la 
structure poteau-poutre en béton, en expérience kaléidoscopique. Cette multiplicité d'images cadrées, mouvantes avec le déplacement du spectateur, est un constant passage d'images cadrées à des "décadrages » et à des "recadrages ». Au lieu d'avoir une "pensée abstraite » du mémorial (le Jardin du souvenir a une fonction de mémoire, comme son nom l'indique clairement), c'est une pensée de l'expérience de l'enchaînement des points de vue que nous pouvons développer, comme c'était le cas pour les trois stations du mémorial Passages. C'est Richard Serra qui fait cette distinction entre "pensée abstraite" et "pensée de l'expérience» dans ses Écrits (Serra, 1973, p. 22). Il décrit l'expérience de surplomb de sa sculpture Shift en ces termes : "Changer le contenu de la perception en faisant coexister le spectateur et la sculpture dans le même espace comportemental. Cela implique mouvement, temps, [et] anticipation. » (Serra, 1983, p. 215.) Ces écrits de Serra font d'ailleurs encore écho à la pensée de Benjamin sur l'expérience, et nous reviendrons sur cette question un peu plus loin.

19 Les plans de Serra éveillent notre conscience de l'espace et du temps, et les cadres du Jardin du souvenir éveillent une conscience plus aiguë du mouvement et du temps, en particulier du présent de notre situation, à travers le filtre du passé. Ces multiples cadres "historiques", par lesquels on cadre des environnements présents, donnent une expérience assimilable aux "constellations » signifiantes d'images décrites par Walter Benjamin. Chez ce dernier, la multiplicité des images, comme autant de cristallisations de moments affectifs et de métaphores de la culture du temps, rend possible le déchiffrement de l'histoire (Benjamin, 2009; voir à ce sujet Palmier, 2006, p. 451). Même si les images qui s'imposent à l'observateur par les cadres de la structure de béton sont fortuites, incontrôlées, que ce soit par Karavan ou par le spectateur, la multiplicité des cadres entraîne un jeu sur le sens des relations entre les différentes « images» créées et produit donc un imaginaire de cette multiplicité d'images. Chez Benjamin, la configuration spatiale est inséparable d'un rapport au temps. La continuité du temps est brisée par sa projection dans un espace éclaté en images qui deviennent autant de symboles et de prophéties. Cette spatialisation permanente du temps - saisi à travers un monde de signes et de hiéroglyphes - constitue le cœur de la plupart des images d'Enfance berlinoise (Palmier, 2006, p. 465). Le passé et le présent ${ }^{11}$ interfèrent, non dans une simple superposition mais dans une tension extrême. L'image dialectique procède d'un arrêt du cours de l'histoire : «Lorsque la pensée s'immobilise dans une constellation saturée de tensions... » (Benjamin, 2009, p. 494 ; à ce sujet, voir Palmier, 2006, p. 467). «Il ne faut pas dire que le passé éclaire le présent ou que le présent éclaire le passé. Une image, au contraire, est ce en quoi l'Autrefois rencontre le Maintenant dans un éclair pour former une constellation. » (Benjamin, 2009, p. 478.) Pour Benjamin, c'est lorsque le présent pénètre par effraction dans cette constellation saturée de tensions que se produit le choc qui permet la cristallisation de pensées. C'est comme un "arc tendu entre le passé et le maintenant, la perdition du sens et son sauvetage » (Palmier, 2006, p. 468). Le présent est alors vécu comme « à-présent », dans lequel se sont fichés des éclats du temps passé12. Le sens temporel de l'installation de Karavan s'inspire en quelque sorte de la philosophie de Benjamin, qu'il a lue avant de travailler au mémorial.

De manière générale, l'esthétique de ces cadres se rapproche des études sur le sens temporel des montages au cinéma (développées par les avant-gardes contemporaines de Benjamin et théorisées par Siegfried Kracauer par exemple) ou des installations vidéo multiécran. Les cadres développent ce sens temporel issu du «collage » de 
cadres, et donc du «montage» des séquences de paysage perçues. Mais ces rapprochements nécessiteraient une étude approfondie. Notons que le montage littéraire est décrit et pratiqué comme méthode d'historien par Benjamin. «La première étape [...] consistera [...] à édifier les grandes constructions à partir de très petits éléments confectionnés avec précision et netteté. Elle consistera même à découvrir dans l'analyse du petit moment singulier le cristal de l'événement total.» (Benjamin, 2009, p. 477.) Cet ouvrage, Le Livre des passages, reste inachevé, et ne nous est d'ailleurs parvenu que par fragments (Guéry, 2009). Il y a chez Benjamin un « aspect pédagogique de ce dessein: "Éduquer en nous l'élément créateur d'images pour lui apprendre à voir de façon stéréoscopique et dimensionnelle dans la profondeur des ombres historiques." La formule est due à Rudolf Borchardt.» (Borchardt, 1923 ; Benjamin, 2009, p. 474.)

21 Cet objectif pédagogique, Dani Karavan le partage également, de la manière dont nous décrivions son travail, notamment par sa pensée optimiste quant à l'impact social et politique potentiel de ses sculptures. La spécificité de la démarche de Karavan est de se situer le plus souvent dans l'espace public. Le fait de travailler dans et avec l'espace public témoigne d'une forme de combat (une forme de militantisme intégrée à sa pratique artistique) pour l'expérience au niveau collectif, ce qui entre également en résonance avec les théories de Benjamin. Pour ce dernier, le dépérissement de l'expérience sur le plan collectif est associé au repli sur la sphère privée : par exemple, dans le domaine de l'écrit, par la disparition du récit au profit du roman et de la solitude du lecteur. Ces mutations trouvent leur équivalent dans la manière de vivre l'architecture et la ville par la transformation de l'expérience (Erfahrung) en expérience vécue (Erlebnis), dans le repli sur la sphère du privé et la transformation, à travers le passage, de la rue en intérieur.

L'engagement théorique et politique de Walter Benjamin culmine dans sa conception du «monde éveillé », avec le passage constant de l'individuel au collectif (Palmier, 2006, p. 448). Cette conception de l'art et de la sculpture n'est pas répandue dans le contexte contemporain. Mais Karavan a su, pour le mémorial Passages et le Jardin du souvenir, renouveler une esthétique du "jardin philosophique», par le moyen des cadres en particulier, dans une esthétique du montage de vues héritée des avantgardes, et dont la contemporanéité est remarquée par Paul Ardenne dans son ouvrage L'Art, le Présent (Ardenne, 2009). L'idée radicale, voire provocatrice, d'évider les bâtiments existants, de faire avec l'existant, dans une esthétique du low, a toute sa place dans les débats artistiques contemporains.

\section{BIBLIOGRAPHIE}

Ardenne, P., L'art, le Présent. La création plasticienne au tournant du XIX ${ }^{e}$ siècle, Paris, Éditions du regard, 2009.

Benjamin, W., «Sur le programme de la philosophie qui vient », CEuvres. Mythe et violence, Denoël, 1971. 
Benjamin, W., « Sur le concept d'histoire », Euvres III (1940), Paris, Gallimard, coll. « Folio Essais », 2000.

Benjamin, W., Paris, capitale du XIX $X^{e}$ siècle. Le Livre des passages, Paris, Les Éditions du cerf, coll.

«Passages », 2009.

Borchardt, R., Epilegoma zu Dante, Paris, Belin, 1923, t. I.

Gloor Fadel, S., Berlin Cinéma (titre provisoire), film, 1997.

Guéry, F., « Art architecture paysage » sur le mémorial Passages, étude réalisée sous la direction de Bruno Queysanne, ministère de la Culture et de la Communication, 2009.

Kimmel, L., « Passages de Dani Karavan : Le "décadrage” comme mémoire du cadre sur le paysage », colloque «Cadrages et paysage », organisé par l'université Paris I La Sorbonne, Centre Saint-Charles, Paris, 2009.

Palmier, J.-M., Walter Benjamin. Le chiffonnier, l'Ange et le Petit Bossu, Paris, Klincksieck, 2006.

Roger, A., Court Traité du paysage, Paris, Gallimard, coll. « Bibliothèque des sciences humaines », 1997.

Serra, R., « Shift » (1973), dans Arts magazine, avril 1973 et dans Écrits et Entretiens 1970-1989, Paris, Daniel Lelong, 1990.

Serra, R., « Entretien avec Peter Eisenman » (1983), dans Skyline. The architecture and design review, New York, avril 1983, et dans Ecrits et Entretiens 1970-1989, Paris, Daniel Lelong, 1990.

Tiberghien, G. A, Land Art, Paris, Carré, 1996.

\section{NOTES}

1. Ce phénomène est décrit par Gilles Tiberghien dans l'ouvrage Land art (Tiberghien, 1996).

2. En plein désert israélien, aux environs de Beersheba, Dani Karavan réalise entre 1963 et 1968 le Monument du Negev, véritable village de sculpture en béton en hommage à la participation de la brigade du Néguev à la guerre d'indépendance d'Israël.

3. Entretien de l'auteure avec Dani Karavan, novembre 2009.

4. Dans le passage de la couleur au noir et blanc, Wim Wenders voit l'« idée qui prime sur la matière » (dans le film de Gloor Fadel, Berlin Cinéma, 1997).

5. Ibid.

6. Propos rapportés par Dani Karavan, novembre 2009.

7. Entretien de l'auteure avec Dani Karavan, novembre 2009.

8. Philosophe allemand, né en 1892 à Berlin, mort à l'âge de 48 ans le 26 septembre 1940 à Portbou, où il a été enterré.

9. Mots inscrits sur la plaque de verre en bas du corridor, nous traduisons.

10. Texte allemand; nous traduisons.

11. Il faut entendre par "présent» l'actualité la plus proche et la plus essentielle, le «maintenant » chargé de contradictions et d'espoirs. Par une telle définition, Benjamin sépare radicalement sa conception de l'image dialectique de l'archétype archaïque et ahistorique jungien, simple morceau de préhistoire, de mythologie primitive, et de l'image surréaliste, purement esthétique où l'histoire est présente de manière passive. L'image surréaliste, chez Aragon en particulier, lui semble encore trop proche du rêve (Benjamin, 2009, p. 474) et prisonnière d'une mythologie. Il affirme au contraire la nécessité de dissoudre la mythologie 
«dans l'espace de l'histoire». À la conception husserlienne des essences, il opposera pareillement la « marque historique » de l'image dialectique (Benjamin, 2009, p. 479-480).

12. "Appendice - L'historicisme se contente d'établir un lien causal entre divers moments de l'histoire. Mais aucune réalité de fait ne devient, par sa simple qualité de cause, un fait historique. Elle devient telle, à titre posthume, sous l'action d'événements qui peuvent être séparés d'elle par des millénaires. L'historien qui part de là cesse d'égrener la suite des événements comme un chapelet. Il saisit la constellation que sa propre époque forme avec telle époque antérieure. Il fonde ainsi un concept du présent comme "à-présent", dans lequel se sont fichés des éclats du temps messianique. » (Benjamin, 1940, p. 443.)

\section{RÉSUMÉS}

Le travail de sculpture mis en œuvre par Dani Karavan pour le Jardin du souvenir à Duisburg consiste en un évidemment de la matière de bâtiments existants, de manière à ne maintenir que la structure, qu'il fait peindre en blanc. Ces éléments, rendus abstraits, ont une fonction perceptive de cadres tridimensionnels sur l'environnement. Les multiples cadres créent autant d'images qui nous font vivre une «constellation » signifiante, en référence à Walter Benjamin. Karavan connaît notamment la philosophie de Benjamin après avoir construit un mémorial en son honneur à Portbou (Espagne) entre 1991 et 1994. Nous nous interrogerons sur l'ancrage de cette expérience dans une esthétique du XIX ${ }^{\mathrm{e}}$ siècle et sur sa contemporanéité, comme piste pour la construction de perceptions par une multiplicité de cadrages et d'images.

Dani Karavan's sculpture work for the Garden of Memories in Duisburg involves taking apart the existing buildings to maintain only their structure, which is painted in white. This process gives the structures an abstract quality, allowing them to be perceived as a three-dimensional framing of the environment. The multiple frames create many images, producing the effect of a signifying "constellation," in reference to Walter Benjamin. Karavan became familiar with Benjamin's philosophy, while creating a memorial in his honor in Portbou (Spain) in 1991-94. We will examine the foundations of this experience within the aesthetics of the 19th century as well as its contemporaneity, as a pathway for the construction of perceptions through multiple frames and images.

\section{INDEX}

Keywords : Dani Karavan, sculpture, Garden of Memories, frame, Walter Benjamin

Mots-clés : Dani Karavan, sculpture, Jardin du souvenir, cadre, Walter Benjamin

\section{AUTEUR}

\section{LAURENCE KIMMEL}

Laurence Kimmel est architecte, docteur en esthétique de l'université Paris X Nanterre, enseignante-chercheuse à l'École nationale supérieure du paysage de Versailles et à l'École 
nationale supérieure d'architecture de Normandie (Rouen).

l.kimmel[at]free[dot]fr 